\title{
Theory of two-photon processes in quantum dots: coherent evolution and phonon-induced dephasing
}

\author{
Paweł Machnikowsk: \\ Institute of Physics, Wroctaw University of Technology, 50-370 Wroctaw, Poland
}

\begin{abstract}
The paper discusses coherent control of charge and spin states of a biexciton system in a quantum dot via coherent two-photon transitions. Rabi oscillations between the ground state of a quantum dot and the biexciton state, as well as oscillations between the two single exciton states induced by laser pulses with different circular or linear polarizations are studied. The effect of phonon-induced decoherence on these processes is described. System properties and driving conditions that lead to optimal coherent control are identified. It is shown that proper optimization allows one to control the two-qubit biexciton system via two-photon transitions with a high fidelity.
\end{abstract}

PACS numbers: 78.67.Hc,42.50.Hz,71.38.-k,03.65.Yz

\section{INTRODUCTION}

Developing efficient methods for optical control of the quantum states of carriers confined in semiconductor nanostructures is not only important from a purely scientific point of view but also vital for emerging technologies. Quantum dots (QDs) have been proposed as an efficient source of on-demand entangled photons $\underline{\underline{1}}$. This application was experimentally realized using optically driven QD systems ${ }^{2}$. Demonstration of Rabi oscillations of the exciton occupation ${ }^{3}$ and conditional control of the biexciton system (two confined excitons with opposite polarizations $)^{\underline{4}}$ demonstrate the feasibility of coherent optical control of charge (orbital) degrees of freedom. Although this suggests that implementing quantum bits on such charge states might be possible the relatively short lifetime of charge excitations considerably restricts the feasibility of this solution. Instead, spin degrees of freedom, which are stable over much longer time scales, are believed to be much more promising $\underline{5}$. Here, again, optical methods allow one to control the system on picosecond time scales, that is, much faster than by electron spin resonance or by electric gating using exchange interaction. A whole range of theoretical proposals for such optical spin control schemes $6.7,8,9$ was recently followed by an experimental demonstration $\underline{10}$. It has also been shown that entanglement generating two-qubit gates can be performed optically on confined spin qubits via various all-optical schemes 11,12 .

While the atomic-like discrete properties of optical transitions in QDs allow one, in principle, to implement a wide variety of quantum optical schemes, the solid state nature of the system introduces various decoherence channels that cannot be ignored when designing optical control schemes. One of the most important sources of dephasing in coherently driven systems is the coupling to lattice degrees of freedom (phonons) $13,14,15$ which imposes additional constraints on the implementation of optical control protocols $\frac{16}{}$. Typically, due to the dynamical character of the lattice response and to the highly structured nature of the lattice reservoir, the requirement of avoiding strong lattice response restricts the parameters of the control fields to a narrow range which can only be determined by careful modelling of the control procedure in the presence of decoherence $\frac{17,18}{}$.

In order to achieve fast control with high fidelity and to provide enough flexibility for optimizing against decoherence processes, new control schemes are sought for. This paper is devoted to a specific class of such schemes: quantum control of a four-level biexciton system using coherent two-photon transitions. The direct motivation for the present study are recent experiments in which coherent two-photon transitions between the ground state and the biexciton state have been demonstrated 19 and full pulse-area-dependent two-photon Rabi oscillations between these two states were induced 20 . Here, a complete theory of coherent two-photon processes in a biexciton system in a QD will be presented. Such processes provide an additional degree of control of the biexciton, which can be viewed as the simplest semiconductor twoqubit system. They can be used to create entanglement with a single laser pulse, which is a valuable alternative to the schemes based on sequential transitions or two-color control. From the spin-oriented point of view, some of the transitions to be described here consist in a simultaneous flip of the electron and hole spin. Both the coherent evolution in an optically driven four-level system and the effect of carrier-phonon coupling will be discussed. It will be shown that QDs with various spectral properties (positive vs. negative biexciton shifts) and under various driving conditions (sign and value of the frequency detuning of the laser field) are optimal for different control schemes.

The paper is organized as follows. Section III introduces the model of the system under study. Next, in Sec. III, the perfect system evolution (without dephasing) is discussed, first for linearly polarized laser pulses (Sec. IIIA), then for circularly polarized ones (Sec. IIIB). Section IV outlines the numerical and analytical (perturbative) methods used for the study of the phonon impact on the system evolution. In Sec. V the system evolution including phonon-induced dephasing is discussed for various system configurations. Sec. VI concludes the paper with a summary and final remarks. In addition, the influ- 

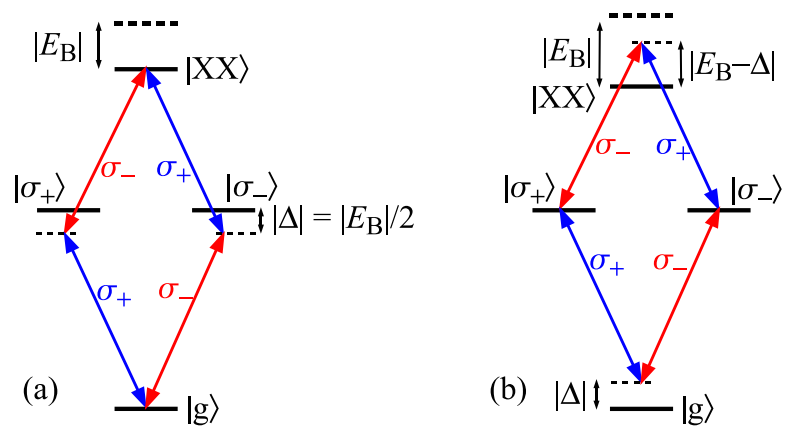

FIG. 1: The energy level structure of a confined biexciton and the schematic presentation of the optical couplings between the states for the biexciton Rabi oscillations (a) and for the exciton spin flip (b).

ence of the exchange splitting of the single exciton states is discussed in the Appendix.

\section{THE SYSTEM}

The system under consideration is composed of a single QD coupled to a laser beam which may have either linear or circular polarization. According to the selection rules, a circularly right-polarized beam can only induce transitions from the ground state $|\mathrm{g}\rangle$ to the right circularly polarized exciton state $\left|\sigma_{+}\right\rangle$and from the left circularly polarized exciton state $\left|\sigma_{-}\right\rangle$to the biexciton state $|\mathrm{XX}\rangle$ (blue arrows labeled ' $\sigma_{+}$' in Fig. 11). The other two transitions (red arrows labeled ' $\sigma_{-}$' in Fig. 1) are allowed for a left-polarized beam. A linearly polarized beam is a superposition of both circularly polarized components and couples both exciton states with fixed circular polarizations $\left(\left|\sigma_{+}\right\rangle,\left|\sigma_{-}\right\rangle\right)$to the ground and biexciton states $(|\mathrm{g}\rangle,|\mathrm{XX}\rangle)$. In this way, the system is modeled by a fourlevel 'diamond' structure, which is a generalization of ' $\Lambda$ systems' and 'V systems' studied in quantum optics.

It is assumed that the frequency of the beam is detuned from all the single-photon transitions, as shown in Fig. 11 For pulses of picosecond durations, the excited states of confined carriers are irrelevant and may be disregarded. In most of the discussion, we will also neglect the exchange interaction that couples the two circularly polarized exciton states and turns them into a weakly split linearly polarized doublet $\underline{21,22,23}$. The energy of this coupling is usually below $100 \mu \mathrm{eV}$ and can be reduced or even cancelled by applying external fields 24,25 or by special treatment (annealing) of the samples ${ }^{26,27}$. In most cases, the small fine structure splitting does not affect the system dynamics on the picosecond time scales relevant for the present discussion. However, it becomes important if the single exciton states are coupled by long (spectrally selective) pulses, so that the fine structure becomes spectrally resolved. This effect is discussed in the Appendix. The effects of finite exciton lifetime (of the order of $1 \mathrm{~ns}$ ) will also be disregarded.
Thus, the system is described by the Hamiltonian

$$
\tilde{H}=\tilde{H}_{\mathrm{XX}}+\tilde{H}_{\mathrm{las}}+H_{\mathrm{ph}}+H_{\mathrm{int}} .
$$

Here, the first term describes the four-level biexciton system,

$$
\tilde{H}_{\mathrm{XX}}=E\left(\left|\sigma_{+}\right\rangle\left\langle\sigma_{+}|+| \sigma_{-}\right\rangle\left\langle\sigma_{-}\right|\right)+\left(2 E+E_{\mathrm{B}}\right)|\mathrm{XX}\rangle\langle\mathrm{XX}|,
$$

where $E$ is the energy of the single exciton states and $E_{\mathrm{B}}$ is the biexciton shift (assumed negative in the binding case, as in Fig. 11). The second term accounts for the coupling between the carrier states and the laser beam. For a linear polarization it has the form

$$
\tilde{H}_{\text {las }}^{(\text {lin })}=f(t) \cos (\Omega t)[(|\mathrm{g}\rangle+|\mathrm{XX}\rangle)\langle\mathrm{X}|+\text { H.c. }],
$$

while for a circular (say, $\sigma_{+}$) polarization it reads

$$
\tilde{H}_{\text {las }}^{(\text {circ })}=f(t) \cos (\Omega t)\left(|\mathrm{g}\rangle\left\langle\sigma_{+}|+| \sigma_{-}\right\rangle\langle\mathrm{XX}|+\text { H.c. }\right),
$$

where $f(t)$ is the envelope of the laser pulse amplitude (which is assumed to be real), $\Omega$ is the laser frequency and we define 'linearly polarized' exciton states $|\mathrm{X}\rangle,|\mathrm{Y}\rangle$, related to the circularly polarized states (i.e., angular momentum eigenstates) by $\left|\sigma_{ \pm}\right\rangle=(|\mathrm{X}\rangle \pm i|\mathrm{Y}\rangle) / \sqrt{2}$. The third term in Eq. (11) is the free phonon Hamiltonian,

$$
H_{\mathrm{ph}}=\sum_{\boldsymbol{k}} \hbar \omega_{\boldsymbol{k}} b_{\boldsymbol{k}}^{\dagger} b_{\boldsymbol{k}}
$$

where $b_{\boldsymbol{k}}^{\dagger}, b_{\boldsymbol{k}}$ are creation and annihilation operators for a phonon with a wave vector $\boldsymbol{k}$ and $\omega_{\boldsymbol{k}}$ is the corresponding frequency. The last term describes carrier-phonon interaction,

$$
\begin{aligned}
& H_{\mathrm{int}}= \\
& \quad(|\mathrm{X}\rangle\langle\mathrm{X}|+| \mathrm{Y}\rangle\langle\mathrm{Y}|+2| \mathrm{XX}\rangle\langle\mathrm{XX}|) \sum_{\boldsymbol{k}} g_{\boldsymbol{k}}^{*}\left(b_{\boldsymbol{k}}+b_{-\boldsymbol{k}}^{\dagger}\right),
\end{aligned}
$$

where the coupling constants $g_{\boldsymbol{k}}=g_{-\boldsymbol{k}}^{*}$ account for the deformation potential coupling between the longitudinal acoustic phonons and a confined neutral exciton. This coupling dominates over the piezoelectric coupling to the acoustic phonon branches if the electron and hole wave functions overlap strongly 28 . On the other hand, coupling to optical phonons is not important in the present study since both the typical frequencies of the system evolution and the magnitudes of the detunings are much lower than the frequencies of the optical phonons.

For the pulse envelope, a Gaussian shape will be assumed,

$$
f(t)=\frac{\hbar \theta}{\sqrt{2 \pi} \tau_{0}} e^{-\frac{1}{2}\left(\frac{t}{\tau_{0}}\right)^{2}},
$$

where $\tau_{0}$ is a parameter defining the pulse duration and

$$
\theta=\int_{-\infty}^{\infty} d t \frac{f(t)}{\hbar}
$$


is the pulse area.

Assuming Gaussian wave functions and neglecting the small correction resulting from the different localization widths of electrons and holes, the coupling constants are given by 29

$$
g_{\boldsymbol{k}}=\left(\sigma_{\mathrm{e}}-\sigma_{\mathrm{h}}\right) \sqrt{\frac{\hbar k}{2 \rho v c}} e^{-l^{2}\left(k_{x}^{2}+k_{y}^{2}\right) / 4-l_{z}^{2} k_{z}^{2} / 4},
$$

where $\sigma_{\mathrm{e}, \mathrm{h}}$ are the deformation potential constants for electrons and holes, $\rho$ is the crystal density, $v$ is the normalization volume for phonon modes, $c$ is the speed of sound, and $l, l_{z}$ are the confinement lengths in the QD plane and along the growth direction. The values used in the calculations are $\sigma_{\mathrm{e}}-\sigma_{\mathrm{h}}=9 \mathrm{eV}, \rho=5350 \mathrm{~kg} / \mathrm{m}^{3}$, $c=5150 \mathrm{~m} / \mathrm{s}, l=4.5 \mathrm{~nm}$, and $l_{z}=2 \mathrm{~nm}$.

The Hamiltonian is transformed to the 'rotating frame' by the canonical transformation defined by the unitary operator

$$
U=e^{i \Omega t\left(\left|\sigma_{+}\right\rangle\left\langle\sigma_{+}|+| \sigma_{-}\right\rangle\left\langle\sigma_{-}|+2| \mathrm{XX}\right\rangle\langle\mathrm{XX}|\right)-i(\Delta / \hbar) t \mathbb{I}},
$$

where $\Delta=\hbar \Omega-E$ is the detuning between the laser frequency and the single exciton transition energy and $\mathbb{I}$ is the identity operator. The transformed Hamiltonian is

$$
H=U H U^{\dagger}+i \hbar \frac{d U}{d t} U^{\dagger}=H_{\mathrm{XX}}+H_{\mathrm{las}}+H_{\mathrm{ph}}+H_{\mathrm{int}},
$$

where

$$
\begin{aligned}
H_{\mathrm{XX}}= & \Delta|\mathrm{g}\rangle\left\langle\mathrm{g}\left|+\left(E_{\mathrm{B}}-\Delta\right)\right| \mathrm{XX}\right\rangle\langle\mathrm{XX}| \\
H_{\text {las }}^{(\text {lin })}= & f(t) \cos (\Omega t) \\
& \times\left[\left(e^{-i \Omega t}|\mathrm{~g}\rangle+e^{i \Omega t}|\mathrm{XX}\rangle\right)\langle\mathrm{X}|+\text { H.c. }\right] \\
H_{\text {las }}^{(\text {circ })}= & f(t) \cos (\Omega t) \\
& \times\left[e^{-i \Omega t}\left(|\mathrm{~g}\rangle\left\langle\sigma_{+}|+| \sigma_{-}\right\rangle\langle\mathrm{XX}|\right)+\text { H.c. }\right]
\end{aligned}
$$

and the contributions $H_{\mathrm{ph}}$ and $H_{\text {int }}$ remain unchanged.

Since the dynamics induced by the laser pulse is slow compared to the frequency of the optical field it is possible to treat the system in the rotating wave approximation ${ }^{30}$, as is commonly done in the description of picosecond dynamics induced by laser fields in the optical or near-infrared range (that is, with femtosecond oscillation periods). One neglects in Eqs. (2) and (3) the very quickly oscillating terms containing $\exp ( \pm 2 i \Omega t)$ which average to null over a typical time scale characterizing the evolution of the system state. This leads to the final formulas for the exciton-laser coupling Hamiltonians

$$
H_{\mathrm{las}}^{(\mathrm{lin})}=\frac{1}{2} f(t)[(|\mathrm{g}\rangle+|\mathrm{XX}\rangle)\langle\mathrm{X}|+\text { H.c. }],
$$

and

$$
H_{\text {las }}^{(\text {circ })}=\frac{1}{2} f(t)\left(|\mathrm{g}\rangle\left\langle\sigma_{+}|+| \sigma_{-}\right\rangle\langle\mathrm{XX}|+\text { H.c. }\right) .
$$
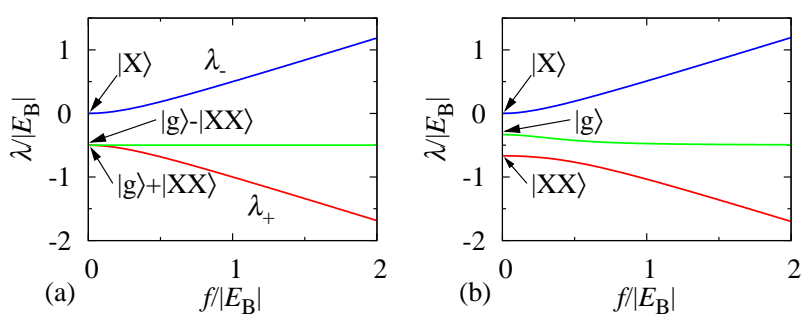

FIG. 2: The spectral branches representing the instantaneous eigenstates of the unperturbed Hamiltonian $H_{\mathrm{XX}}+H_{\text {las }}^{((\operatorname{lin}))}$ as functions of the pulse amplitude $f$ (the adiabatic parameter) for $\Delta=E_{\mathrm{B}} / 2$ (a) and for $\Delta=E_{\mathrm{B}} / 3$ (b). In both cases, $E_{\mathrm{B}}<0$.

\section{UNPERTURBED EVOLUTION}

In this section, various schemes of two-photon coherent control of a biexciton system are discussed on a purely quantum-optical level, that is, without phonon-induced dephasing. The optically induced transitions depend on the polarization of the laser pulse. The following two subsections are devoted to the evolution driven by linearly and circularly polarized laser pulses, respectively.

\section{A. Linear polarization}

Let us start with the unperturbed evolution of the system, generated by the Hamiltonian $H_{\mathrm{Xx}}+H_{\text {las }}^{(\text {lin) }}$ [Eqs. (1) and (4)]. Consider the diagram of instantaneous eigenstates of the relevant three-level Hamiltonian (excluding the decoupled state $|Y\rangle$ ) as a function of the pulse amplitude $f$. First, let us focus on the special case of $\Delta=E_{\mathrm{B}} / 2$ [Fig. 2(a)]. Then

$$
\left.H_{\mathrm{XX}}=\frac{\Delta}{2}(|+\rangle+|+|-\rangle-\mid\right),
$$

where $| \pm\rangle=(|\mathrm{g}\rangle \pm|\mathrm{XX}\rangle) / \sqrt{2}$. The driving field [Eq. (4)] does not couple the state $|-\rangle$ to the other states, hence this state is invariant under $H_{\mathrm{Xx}}+H_{\text {las }}^{(\text {lin) }}$. Another, nontrivial invariant subspace is spanned by the states $|\mathrm{X}\rangle$ and $|+\rangle$. The instantaneous eigenvalues along the two spectral branches belonging to this subspace are

$$
\lambda_{ \pm}(t)=\frac{\Delta}{2}\left\{1 \pm \sqrt{1+2[f(t) / \Delta]^{2}}\right\}
$$

where $\lambda_{-}$corresponds to the branch originating from the state $|\mathrm{X}\rangle$ [upper branch, blue line in Fig. 2(a)]. It is clear that the two branches are separated from each other by at least $|\Delta|$.

If the amplitude $f$ of the laser pulse changes slowly enough in time the evolution generated by $H_{\mathrm{Xx}}$ may be found using the adiabatic theorem 20,31 . In such case, the states $|\mathrm{X}\rangle$ and $|+\rangle$ undergo an adiabatic evolution, with $f(t)$ playing the role of a slowly varying parameter. 
At the time $t_{1}$, after the pulse has been switched off, the initial state is restored with the additional dynamical phase

$$
\alpha_{ \pm}=-\frac{1}{\hbar} \int_{t_{0}}^{t_{1}} d t \lambda_{ \pm}(t)
$$

where $t_{0}$ is the initial time (before the pulse was switched on).

Assume now that the system is initially prepared in the state

$$
\left|\sigma_{+}\right\rangle=\frac{|\mathrm{X}\rangle+i|\mathrm{Y}\rangle}{\sqrt{2}} .
$$

The state $|\mathrm{Y}\rangle$ is decoupled from the laser beam and does not evolve. As a result, the state $\left|\sigma_{+}\right\rangle$undergoes the transformation

$$
\begin{aligned}
\left|\sigma_{+}\right\rangle & \longrightarrow \frac{e^{i \alpha_{-}}|\mathrm{X}\rangle+i|\mathrm{Y}\rangle}{\sqrt{2}} \\
& =e^{i \alpha_{-} / 2}\left(\cos \frac{\alpha_{-}}{2}\left|\sigma_{+}\right\rangle+i \sin \frac{\alpha_{-}}{2}\left|\sigma_{-}\right\rangle\right)
\end{aligned}
$$

The phase $\alpha_{-}$may be arbitrarily large. Moreover, for a fixed pulse shape, it is a monotonous function of the pulse intensity. Thus, by varying the pulse amplitude, the exciton can be coherently rotated between the two polarization states $\left|\sigma_{+}\right\rangle$and $\left|\sigma_{-}\right\rangle$. The resulting occupation of the state $\left|\sigma_{-}\right\rangle$, obtained from numerical solution of the quantum evolution equation, is shown in Fig. 3(a) (red solid line) as a function of the pulse area $\theta$.

In the same way, since the state $|-\rangle$ evolves only trivially, if the system is initially in the state

$$
|\mathrm{g}\rangle=\frac{|+\rangle+|-\rangle}{\sqrt{2}},
$$

it will undergo the transformation

$$
|\mathrm{g}\rangle \longrightarrow e^{i\left[\alpha_{+}-\Delta\left(t_{1}-t_{0}\right) / \hbar\right] / 2}\left(\cos \frac{\beta}{2}|\mathrm{~g}\rangle+i \sin \frac{\beta}{2}|\mathrm{XX}\rangle\right),
$$

where $\beta=\alpha_{+}+\Delta\left(t_{1}-t_{0}\right) /(2 \hbar)$. Note that $\lambda_{+} \rightarrow \Delta$ when the laser pulse is switched off, so that $\beta$ is in fact independent of the choice of the initial and final time. Thus, when the pulse amplitude is increased the system oscillates between the ground and biexciton states, much like in the usual pulse-area dependent Rabi oscillations between the ground and single exciton states, induced by a resonant circularly polarized beam ${ }^{3}$. The biexciton oscillations are plotted in Fig. 3(b) (red solid line). Such oscillations were indeed observed in an experiment ${ }^{20}$.

In spite of some qualitative similarity to the Rabi oscillations in a two-level system, described by the universal function $\sin ^{2}(\theta / 2)$, one can clearly see essential differences. The two-photon oscillations are not strictly periodic, especially for weak pulses, when the transition probability develops very slowly. In fact, for weak pulses the occupation of the other state grows as $\theta^{4} \sim I^{2}$, where
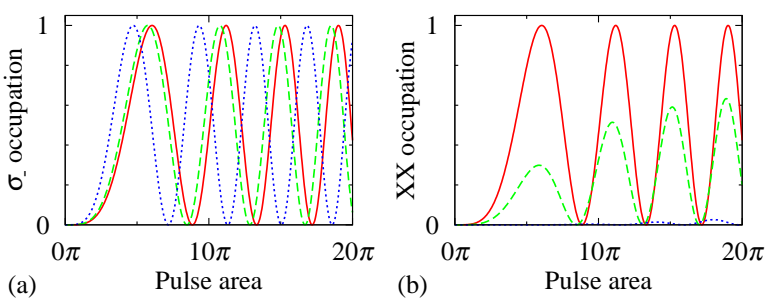

FIG. 3: (a) Two-photon polarization flip: the occupation of the state $\left|\sigma_{-}\right\rangle$as a function of the pulse area for $\Delta=E_{\mathrm{B}} / 2=$ $-2 \mathrm{meV}$ (red solid), $\Delta=E_{\mathrm{B}} / 3$ (green dashed), and $\Delta=$ $E_{\mathrm{B}} / 6$ (blue dotted). (b) Two-photon Rabi oscillations ${ }^{20}$ : the occupation of the biexciton state as a function of the pulse area for $\Delta=E_{\mathrm{B}} / 2=-2 \mathrm{meV}$ (red solid), $\Delta=-1.9 \mathrm{meV}$ (green dashed), and $\Delta=-1.7 \mathrm{meV}$ (blue dotted). In both figures, $E_{\mathrm{B}}=-4 \mathrm{meV}$ and $\tau_{0}=5 \mathrm{ps}$.

$I$ is the pulse intensity, as expected for a two-photon process (see Ref. 20). Moreover, it is clear from Eq. (6) that the rotation angle $\beta$ is a nonlinear functional of the pulse envelope. Hence, contrary to the usual Rabi oscillations, no universal area theorem exists for the final occupations.

In the general case, $\Delta \neq E_{\mathrm{B}} / 2$, there are no invariant states (apart from $|\mathrm{Y}\rangle$ ) and all three states $|\mathrm{g}\rangle,|\mathrm{X}\rangle,|\mathrm{XX}\rangle$ give rise to three non-degenerate spectral branches [Fig. 2(b)]. Now, in the adiabatic limit, the state $|\mathrm{X}\rangle$ evolves as previously, except for a different value of the corresponding adiabatic eigenvalue, which now cannot be given in a simple analytical form. Thus, the exciton spin flip effect will take place also in this case, as shown in Fig. 31(a) (green dashed and blue dotted lines).

On the other hand, since the states $|\mathrm{g}\rangle,|\mathrm{XX}\rangle$ now belong to non-degenerate branches, an adiabatic evolution starting from any of these states will end up in the same state, up to an irrelevant global phase. Therefore, the two-photon Rabi oscillations are suppressed when the driving field is detuned from $\Delta=E_{\mathrm{B}} / 2$, as shown in Fig. 3(b) (green dashed and blue dotted lines). In fact, the symmetric detuning condition is equivalent to the two-photon resonance between the ground and biexciton states. If the two-photon resonance condition $\Delta=E / 2$ is not satisfied, the transition from the ground to biexciton state is forbidden and the corresponding Rabi oscillations are precluded.

On the contrary, the polarization flip is always resonant and the detuning becomes a tunable parameter. As will be shown in the next section, this additional freedom of control may be useful for optimizing experimental parameters against phonon-induced dephasing.

\section{B. Circular polarization}

In the case of a $\sigma_{+}-$polarized beam, the unperturbed system evolution is generated by the Hamiltonian $H_{\mathrm{XX}}+$ $H_{\text {las }}^{\text {(circ) }}$ which has two invariant two-dimensional sub- 


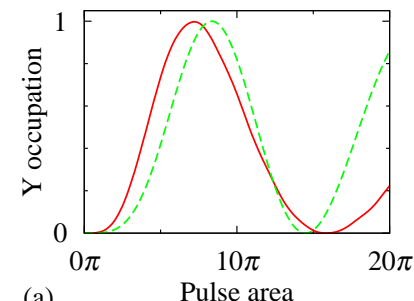

(a)

Pulse area

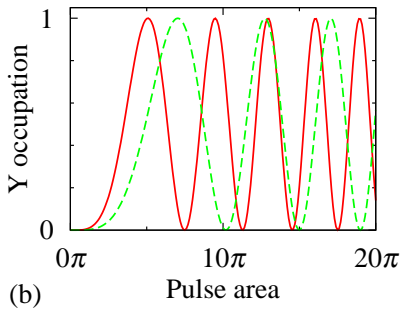

FIG. 4: (Color online) Two-photon rotation of the linear polarization of an exciton state with a circularly polarized pulse: the occupation of the $|\mathrm{Y}\rangle$ state as a function of the pulse area for $E_{\mathrm{B}}=-4 \mathrm{meV}$ and two values of $\Delta:=-3 \mathrm{meV}$ (a) and $+2 \mathrm{meV}$ (b). Red solid lines: $\tau_{0}=5 \mathrm{ps}$, green dashed lines: $\tau_{0}=10$ ps. The initial state is $|\mathrm{X}\rangle$.

spaces spanned, respectively, by the states $|\mathrm{g}\rangle,\left|\sigma_{+}\right\rangle$and $|\mathrm{XX}\rangle,\left|\sigma_{-}\right\rangle$. In the adiabatic limit, each of these four states follows its own branch of instantaneous eigenstates and is restored after the laser is switched off, up to a phase factor. Like in the previously discussed cases, these phase factors can result in a nontrivial evolution if one starts with a system prepared in a superposition of the states. For definiteness, let us choose the initial state

$$
|\mathrm{X}\rangle=\frac{\left|\sigma_{+}\right\rangle+\left|\sigma_{-}\right\rangle}{\sqrt{2}} .
$$

The instantaneous eigenvalues corresponding to the two branches departing from $\left|\sigma_{+}\right\rangle$and $\left|\sigma_{-}\right\rangle$are

$$
\begin{aligned}
& \lambda_{+}=\Delta \frac{1-\sqrt{1+[f(t) / \Delta]^{2}}}{2}, \\
& \lambda_{-}=\left(E_{\mathrm{B}}-\Delta\right) \frac{1-\sqrt{1+\left[f(t) /\left(E_{\mathrm{B}}-\Delta\right)\right]^{2}}}{2} .
\end{aligned}
$$

The final state resulting from the adiabatic evolution is then

$$
|\psi\rangle=e^{i \frac{\alpha_{+}+\alpha_{-}}{2}}\left(\cos \frac{\alpha_{+}-\alpha_{-}}{2}|\mathrm{X}\rangle-\sin \frac{\alpha_{+}-\alpha_{-}}{2}|\mathrm{Y}\rangle\right),
$$

where $\alpha_{ \pm}$is defined by Eq. (7) but with $\lambda_{ \pm}$defined in Eqs. (8a,b). Thus, the polarization of the exciton state can be rotated as long as $\alpha_{+}-\alpha_{-} \neq 0$. This condition is clearly not satisfied at the biexciton resonance, $\Delta=E_{\mathrm{B}} / 2$, but, as long as the laser beam is detuned from this resonance, one expects polarization rotation to appear. This is confirmed by the numerical integration of the evolution equation, as shown in Fig. 4. Note that the final state is always linearly polarized, at an angle of $\left(\alpha_{+}-\alpha_{-}\right) / 2$ to the initial polarization.

\section{PHONON EFFECTS: THE METHOD}

This section presents the general theoretical framework for calculating the effect of the phonon-induced decoherence on the two-photon transitions described above. This will be followed by the results of numerical simulations discussed in the next section.

It is convenient to perform a unitary transformation to the phonon-dressed exciton states 32 ,

$$
\mathbb{W}=|\mathrm{g}\rangle\langle\mathrm{g}|+(|\mathrm{X}\rangle\langle\mathrm{X}|+| \mathrm{Y}\rangle\langle\mathrm{Y}|) W+| \mathrm{XX}\rangle\langle\mathrm{XX}| W^{2}
$$

where

$$
W=\exp \left[-\sum_{\boldsymbol{k}} \frac{g_{\boldsymbol{k}}^{*}}{\hbar \omega_{\boldsymbol{k}}}\left(b_{\boldsymbol{k}}-b_{-\boldsymbol{k}}^{\dagger}\right)\right]
$$

Note that this transformation conserves the occupations of the basis states. Working in the dressed basis physically corresponds to the assumption that the initial state was prepared some time (a few ps) before the operation and it is therefore surrounded by the polaron-like coherent phonon field. Upon this transformation and expansion to the leading order in the coupling constants, the Hamiltonian may be written as $H^{\prime}=\mathbb{W} H \mathbb{W}^{\dagger}=H_{0}+V$, where the the first component in the two cases of linear and circular beam polarization is, respectively,

$$
\begin{aligned}
H_{0}^{(\text {lin })}= & \Delta|\mathrm{g}\rangle\left\langle\mathrm{g}\left|+H_{\mathrm{ph}}+\left(E_{\mathrm{B}}-\Delta\right)\right| \mathrm{XX}\right\rangle\langle\mathrm{XX}| \\
& +\frac{w}{2} f(t)[(|\mathrm{g}\rangle+|\mathrm{XX}\rangle)\langle\mathrm{X}|+\text { H.c. }]
\end{aligned}
$$

and

$$
\begin{aligned}
H_{0}^{(\text {circ })=} & \Delta|\mathrm{g}\rangle\left\langle\mathrm{g}\left|+H_{\mathrm{ph}}+\left(E_{\mathrm{B}}-\Delta\right)\right| \mathrm{XX}\right\rangle\langle\mathrm{XX}| \\
& +\frac{w}{2} f(t)\left(|\mathrm{g}\rangle\left\langle\sigma_{+}|+| \sigma_{-}\right\rangle\langle\mathrm{XX}|+\text { H.c. }\right),
\end{aligned}
$$

while the second term, describing the interaction with the phonon reservoir, can be written as $V=S^{(\text {lin/circ })} \otimes R$, where

$$
S^{(\operatorname{lin})}=-\frac{i}{\sqrt{2}} f(t)|-\rangle \mathrm{X} \mid+ \text { H.c., }
$$

$$
S^{(\text {circ })}=-\frac{i}{\sqrt{2}} f(t)|g\rangle\left\langle\sigma_{+}|+| \sigma_{-}\right\rangle\langle\mathrm{XX}|+\text { H.c. },
$$

and

$$
R=i \sum_{\boldsymbol{k}} \frac{g_{\boldsymbol{k}}^{*}}{\hbar \omega_{\boldsymbol{k}}}\left(b_{\boldsymbol{k}}-b_{-\boldsymbol{k}}^{\dagger}\right)
$$

Here,

$$
w=1-\frac{1}{2} \sum_{k}\left|\frac{g_{k}}{\hbar \omega_{k}}\right|^{2}\left(2 n_{k}+1\right)
$$

where $n_{\boldsymbol{k}}$ is the phonon occupation number, accounts for the phonon-induced renormalization of the pulse amplitude in the slow driving limit 15,33 . We neglect the phonon-induced energy shifts which are very small for acoustic phonons.

The evolution of the reduced density matrix of the biexciton subsystem is found by numerically solving the 
time-convolutionless (TCL) evolution equation ${ }^{34}$ for the density matrix in the interaction picture with respect to $H_{0}$,

$$
\dot{\rho}(t)=-\int_{0}^{t} d \tau \operatorname{Tr}_{\mathrm{ph}}\left[V(t),\left[V(\tau), \rho(t) \otimes \rho_{\mathrm{ph}}\right]\right],
$$

where $V(t)$ is the interaction Hamiltonian $V$ in the interaction picture with respect to $H_{0}, \rho_{\mathrm{ph}}$ is the phonon density matrix at the thermal equilibrium, and $\operatorname{Tr}_{\mathrm{ph}}$ denotes the partial trace over the phonon degrees of freedom.

The quantitative results presented in the following section will be obtained from a numerical solution to Eq. (9). However, much additional insight can be gained from a perturbative approximation to this equation ${ }^{15,35,36}$ and from the spectral interpretation it provides 14,37 . Let us note that, as long as the perturbation to the system evolution remains small, the reduced density matrix in the interaction picture differs little from its initial value. Hence, $\rho(t)$ on the right-hand side of Eq. (9) may be replaced by its initial value $\rho_{0}=\left|\psi_{0}\right\rangle\left\langle\psi_{0}\right|$, where we assume that the initial system state is pure and represented by the state vector $\left|\psi_{0}\right\rangle$. Upon integration, Eq. (9) then yields

$$
\rho(t)=\rho_{0}-\int_{0}^{t} d \tau \int_{0}^{\tau} d \tau^{\prime} \operatorname{Tr}_{\mathrm{ph}}\left[V(\tau),\left[V\left(\tau^{\prime}\right), \rho_{0} \otimes \rho_{\mathrm{ph}}\right]\right] .
$$

As a simple measure of the phonon-induced perturbation we will use the probability that the system in its actual state $U_{0} \rho(t) U_{0}^{\dagger}$ will be found in the desired, unperturbed state $U_{0}\left|\psi_{0}\right\rangle$ (here, $U_{0}$ is the unperturbed evolution generated by $H_{0}$ ). This is equal to $\mathcal{F}^{2}=$ $\left\langle\psi_{0}|\rho(t)| \psi_{0}\right\rangle$, where $\mathcal{F}$ is the fidelity for the special case of a pure state $\frac{38}{}$. It is convenient to write $\mathcal{F}^{2}=1-\delta$, where $\delta$ is referred to as the error of a quantum control operation. A physically transparent and meaningful formula for $\delta$ is obtained by defining the frequency-dependent operator

$$
Y(\omega)=\frac{1}{\hbar} \int_{0}^{t} d \tau S(\tau) e^{i \omega \tau}
$$

and the phonon spectral density

$$
R(\omega)=\frac{\omega^{2}}{2 \pi} \int_{-\infty}^{\infty} d t\langle R(t) R\rangle e^{i \omega t},
$$

where $S(t)$ and $R(t)$ denote the operators in the interaction picture with respect to $H_{0}$. Then, one can write

$$
\delta=\int_{-\infty}^{\infty} d \omega \frac{R(\omega)}{\omega^{2}} \sum_{l} S_{l}(\omega)
$$

where the spectral functions

$$
S_{l}(\omega)=\left|\left\langle\psi_{l}|Y(\omega)| \psi_{0}\right\rangle\right|^{2}
$$

can be identified with different decoherence channels and the summation over $l$ runs through all states $\left|\psi_{l}\right\rangle$ orthogonal to $\left|\psi_{0}\right\rangle$ (see Refs. 17, 18 for details).
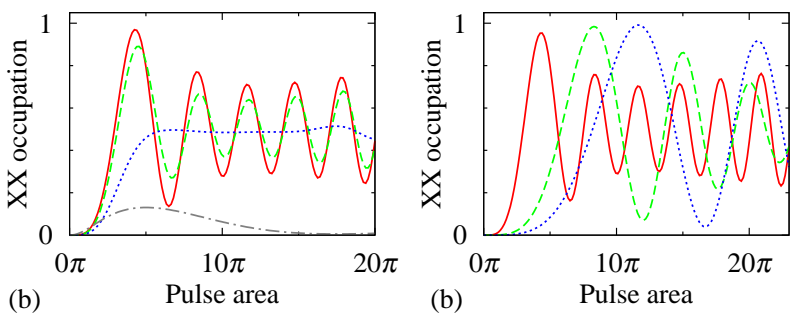

FIG. 5: (Color online) The two-photon Rabi oscillations in the presence of phonon-induced perturbation. (a) The dependence of the damping on temperature for $\tau_{0}=5$ ps: $T=0$ (red solid), $T=10 \mathrm{~K}$ (green dashed), and $T=40 \mathrm{~K}$ (blue dotted). The grey dash-dotted line shows the joint occupation of the single exciton states due to real transitions at $T=40 \mathrm{~K}$. (b) The dependence on the pulse duration at $T=4 \mathrm{~K}: \tau_{0}=5 \mathrm{ps}$ (red solid), $\tau_{0}=20 \mathrm{ps}$ (green dashed) and $\tau_{0}=40 \mathrm{ps}$ (blue dotted).

\section{PHONON EFFECTS: RESULTS}

This section is devoted to the system evolution for different structures of the biexciton spectrum and under various driving conditions. In the first two subsections we will study the phonon impact on the biexciton Rabi oscillations and exciton spin flip induced by a linearly polarized laser field in a system with a negative biexciton shift (bound biexciton). The third subsection contains a discussion of the same processes in a system with antibound biexcitons (a positive biexciton shift) and the last one deals with phonon-induced dephasing in the twophoton rotation of the linear exciton polarization induced by a circularly polarized laser light.

\section{A. Biexciton Rabi oscillations for bound biexcitons}

Let us start the discussion with the case of biexciton oscillations in a system with a negative biexciton shift, $E_{\mathrm{B}}<0$ (bound biexciton). In this case, the laser beam is detuned down from the transition to single exciton states, as shown in Fig. 1(a). Therefore, one can expect that real phonon-assisted transitions to these states will be suppressed at low enough temperatures and the decoherence will be dominated by pure dephasing, like in the two-level case $\frac{14,15}{}$. Indeed, the oscillations presented in Fig. 5(a) show symmetric damping up to temperatures of several Kelvins. Only when $\Delta \sim k_{\mathrm{B}} T$, the phonon-assisted transition up to the single exciton state $|\mathrm{X}\rangle$ is possible. As a result, the damping becomes much stronger and biased towards lower biexciton occupations, which is accompanied by a growing single exciton component, as shown by the grey dash-dotted line in Fig. 5(a), corresponding to $T=40 \mathrm{~K}$.

The dynamical pure dephasing effect is related to the lattice relaxation after a non-adiabatic (with respect to the lattice response times) change of the charge state $\underline{39}$ 

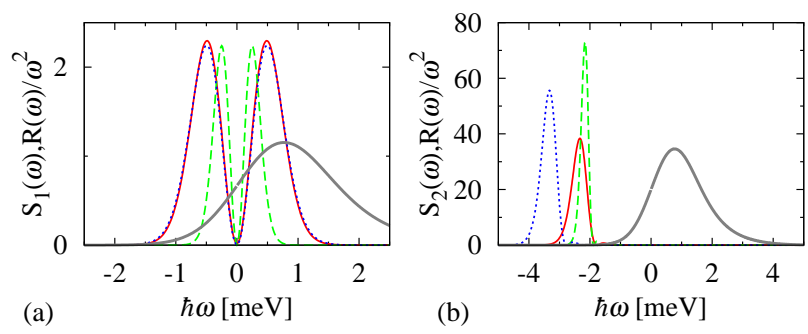

FIG. 6: (Color online) The spectral density of the phonon reservoir at $T=4 \mathrm{~K}$ (thick grey lines on both plots, arbitrarily scaled) and the spectral functions $S_{1}$ (a) and $S_{2}$ (b). Red solid lines: $E_{\mathrm{B}}=-4 \mathrm{meV}, \tau_{0}=5 \mathrm{ps}$; green dashed lines: $E_{\mathrm{B}}=-4 \mathrm{meV}, \tau_{0}=10 \mathrm{ps}$; blue dotted lines: $E_{\mathrm{B}}=-6 \mathrm{meV}$, $\tau_{0}=5$ ps. Here always $\Delta=E_{\mathrm{B}} / 2$.

which correlates the exciton system with the phonon reservoir, generating a kind of "which path" information in the macroscopic environment ${ }^{40}$. Therefore, this kind of decoherence decreases when the optically driven system evolution becomes slower ${ }^{36}$. This behavior is clearly visible in Fig. 5(b).

The properties of the two contributions to dephasing (pure dephasing and real transitions) may be conveniently studied using the approximate perturbative formula (10). Fig. 6] shows the phonon spectral density $R(\omega) / \omega^{2}$ at $T=4 \mathrm{~K}$ and the two spectral functions $S_{i}(\omega)$, with $\left|\psi_{1}\right\rangle=|\mathrm{XX}\rangle$ and $\left|\psi_{2}\right\rangle=|\mathrm{X}\rangle$, for two different pulse durations. In all these plots, the nominal pulse area was tuned to achieve a complete Rabi flop [the first maximum in Fig. 3(b)]. One can see that the first function is always positioned around the zero frequency and its area (hence its overlap with $R(\omega) / \omega^{2}$ ) decreases for slower driving. Therefore, this spectral function may be identified with the pure dephasing effect which decreases for slower driving. The shape of this function does not change considerably when the value of the biexciton shift $E_{\mathrm{B}}$ is altered. On the other hand, the second spectral function is located in the negative frequency area, around $\hbar \omega \approx \Delta$. This allows us to relate this function to the real phonon-assisted transitions to the single exciton state $|X\rangle$. Since the phonon spectral density at large negative frequencies is small at low temperatures this decoherence channel is of minor importance in a system with a large enough biexciton shift and at sufficiently low temperatures.

One notes in Fig. 6(b) that the shape of this spectral feature depends both on $\Delta$ and on $\tau_{0}$. In order to study this real transition process more quantitatively let us note that for a localized spectral function $S_{2}(\omega)$, the corresponding contribution to the error may be approximated by $\delta_{2} \approx \hbar A_{2} R(\Delta) / \Delta^{2}$, where

$$
A_{2}=\hbar \int_{-\infty}^{\infty} d \omega S_{2}(\omega)
$$

The values of $A_{2}$ as a function of the pulse duration $\tau_{0}$ are plotted in Fig. 7(a). Again, for each $\tau_{0}$, the pulse area
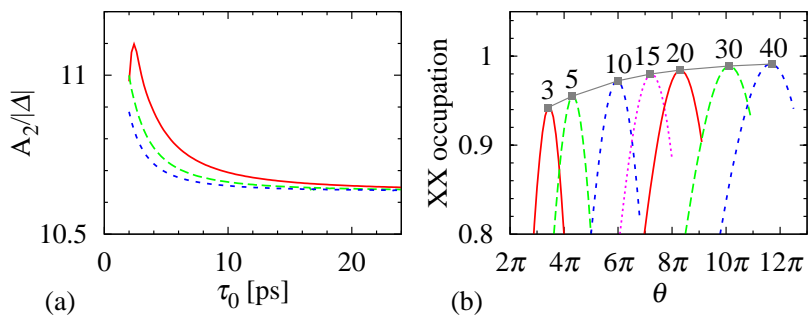

FIG. 7: (Color online) (a) The value of $A_{2} /|\Delta|$ for $E_{\mathrm{B}}=$ $-2 \mathrm{meV}$ (red solid), $E_{\mathrm{B}}=-6 \mathrm{meV}$ (green dashed), and $E_{\mathrm{B}}=$ $-8 \mathrm{meV}$ (blue dotted). Here always $\Delta=E_{\mathrm{B}} / 2$. (b) The first maximum of the biexciton occupation for $\Delta=E_{\mathrm{B}} / 2=$ $-2 \mathrm{meV}, T=4 \mathrm{~K}$, and pulse durations $\tau_{0}$ as indicated. The grey line is added to guide the eye through the maxima of the plotted curves.

is adjusted in order to achieve a complete Rabi flop [the first maximum of the biexciton occupation in Fig. [3(b)], so that all the points correspond to the same transformation of the system state. The overall probability of a transition to the single exciton state depends not only on the duration of the process but also on the occupation of the single exciton state during the evolution. The latter decreases for longer and weaker pulses. It turns out that the two effects compensate each other almost exactly, leading to a nearly constant value of $A_{2}$ as $\tau_{0}$ is varied. This means that the error resulting from the real transition process is constant and, consequently, the biexciton occupation should be achieved with a constant (independent of $\tau_{0}$ ) accuracy as soon as the evolution is slow enough for the pure dephasing to be negligible. This is confirmed by the numerical solution of the TCL equation (9), presented in Fig. 7(b). An additional property, clearly seen in Fig. 7(a), is that the value of $A_{2}$ is nearly exactly proportional to the detuning $\Delta$, with just a small variation (a few per cent) for very short pulses.

\section{B. Exciton spin flip}

The spectral relations are different in the case of a twophoton spin flip. Now, for a negative biexciton energy, the laser frequency is always detuned up from at least one of the transitions [Fig. 1(b)]. For the detuning as in the previous case, both transitions involve phonon emission and are therefore expected to cause much decoherence even at low temperatures. Indeed, the oscillations of the exciton spin orientation shown in Fig. 8(a) are damped in an asymmetric way (biased towards lower occupations), which is a signature of occupation leakage out of the twodimensional single exciton subspace. Correspondingly, single exciton occupation becomes non-zero even at zero temperature. Moreover, the degree of decoherence does not decrease with a growing pulse duration [Fig. 8(b)], again showing that the Markovian real transition pro- 

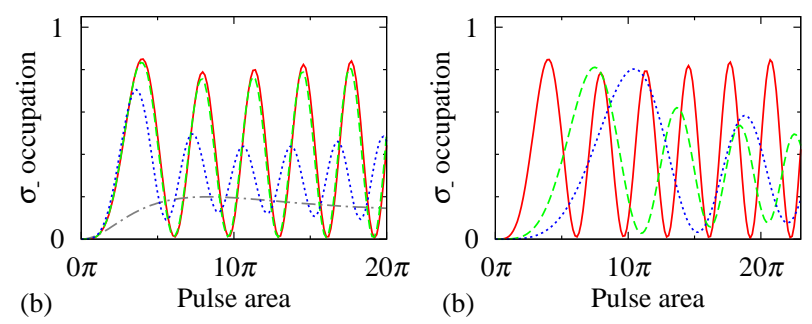

FIG. 8: (Color online) The exciton spin oscillations in the presence of phonon-induced perturbation for $E_{\mathrm{B}}=2 \Delta=$ $-4 \mathrm{meV}$. (a) The dependence of the damping on temperature for $\tau_{0}=5$ ps: $T=0$ (red solid), $T=10 \mathrm{~K}$ (green dashed), and $T=40 \mathrm{~K}$ (blue dotted). The grey dash-dotted line shows the joint occupation of the single exciton states due to real transitions at $T=0 \mathrm{~K}$. (b) The dependence on the pulse duration at $T=4 \mathrm{~K}: \tau_{0}=5 \mathrm{ps}$ (red solid), $\tau_{0}=20 \mathrm{ps}$ (green dashed) and $\tau_{0}=40 \mathrm{ps}$ (blue dotted).
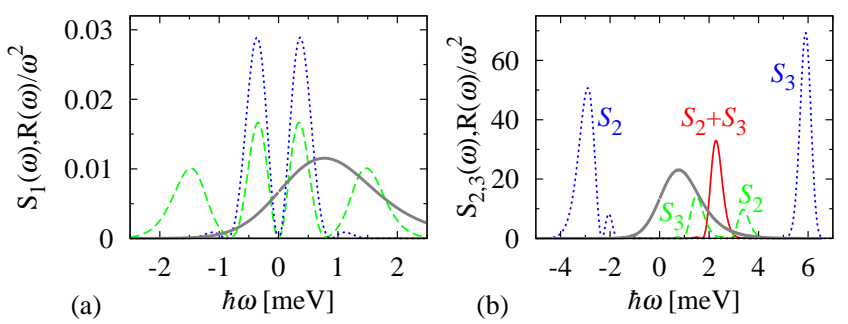

FIG. 9: (Color online) The spectral density of the phonon reservoir at $T=4 \mathrm{~K}$ (thick grey lines on both plots, arbitrarily scaled) and the spectral functions $S_{1}$ (a) and $S_{2}, S_{3}$ (b), for $\Delta=-2 \mathrm{meV}$ (solid red), $\Delta=-3 \mathrm{meV}$ (green dashed), and $\Delta=+2 \mathrm{meV}$ (blue dotted). In all the cases, $E_{\mathrm{B}}=-4 \mathrm{meV}$ and $\tau_{0}=5 \mathrm{ps}$.

cesses are a non-negligible factor.

Interestingly, comparing Fig. 8 with Fig. 5 one can see that the overall dephasing of the two-photon spin flip is weaker than that of the biexciton oscillations, except for the first maximum at low temperatures. The reason for this is that the carrier-phonon interaction is insensitive to the spin orientation, hence there is no phonon-response to the transition between the two single exciton states. There could be some perturbation resulting from the occupation of the other two states during the evolution but in the case of symmetric detuning, $\Delta=E_{\mathrm{B}} / 2$, both these occupations are equal and the average charge confined in the dot remains constant, which precludes any phonon response that might lead to pure dephasing. The spectral function related to the pure dephasing process is again $S_{1}(\omega)$, corresponding to $\left|\psi_{1}\right\rangle=\left|\sigma_{+}\right\rangle$in Eq. (12). In the present case, this function remains null. This changes slightly if the detunings from the ground and biexciton states are not equal and, consequently, there is a charge variation during the evolution. However, as shown in Fig. 9, even in this case the spectral function $S_{1}(\omega)$ is
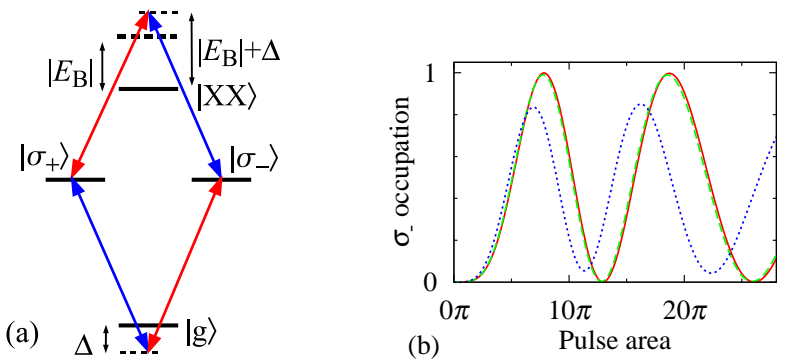

FIG. 10: (Color online) (a) The diagram of energy levels and laser frequencies in the case of $\Delta>0, E_{\mathrm{B}}<0$. (b) The twophoton exciton spin oscillations in the presence of phononinduced perturbation for $\Delta>0, E_{\mathrm{B}}<0$ for $\tau_{0}=5$ ps: $T=0$ (red solid), $T=10 \mathrm{~K}$ (green dashed), and $T=40 \mathrm{~K}$ (blue dotted).

many orders of magnitude smaller than in the case of biexciton oscillations [compare Fig. 9(a) with Fig. 6(a)].

Phonon-assisted real transitions to the ground and biexciton states are now described by two spectral functions $S_{2}(\omega)$ and $S_{3}(\omega)$, corresponding to $\left|\psi_{2}\right\rangle=|\mathrm{g}\rangle$ and $\left|\psi_{3}\right\rangle=|\mathrm{XX}\rangle$ in Eq. (12), respectively. As can be seen in Fig. 9(b), these spectral features shift according to the detuning between the laser frequency and the relevant states.

The fact that the spin flip does not require the twophoton resonance allows one to use the detuning $\Delta$ as a free parameter to optimize the control conditions against the phonon-induced real transitions. The most favorable choice is to set $\Delta>0$ or $\Delta<E_{\mathrm{B}}$ (still assuming $E_{\mathrm{B}}<0$ ). Then, one of the transitions is detuned down from resonance and involves phonon absorption which makes it ineffective at low enough temperatures [see Fig. 10(a)]. The other transition is detuned up but the detuning can be chosen large enough to be brought beyond the cutoff of the phonon spectral density, as shown in Fig. 9(b) (dotted blue lines). The simulations based on the TCL equation (9), presented in Fig. 10(b), show that spin flipping with fidelities of the order of $10^{-3}$ is possible under such conditions. Note that this is achieved with a relatively short pulse so that the effect of the radiative decay during the operation is also small (of the order of $1 \%$ ).

\section{Antibound biexciton}

Although the unperturbed evolution for $E_{\mathrm{B}}>0$ (antibound biexciton) does not differ from that discussed above, this is no more true for the actual system kinetics in the presence of phonons. The two-photon resonance condition $\Delta=E_{\mathrm{B}} / 2$ that must be satisfied for the two-photon Rabi oscillations to occur now means that the laser beam is detuned up from the single exciton transition. Unless this detuning is large enough (which would require large positive biexciton shifts) phononassisted transitions to the single exciton states may now take place, involving an emission of a phonon and there- 


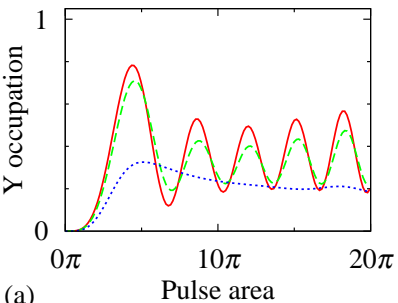

(a)

Pulse area

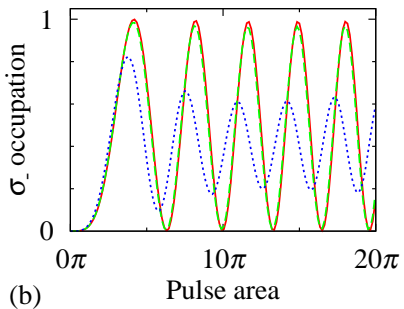

(b)

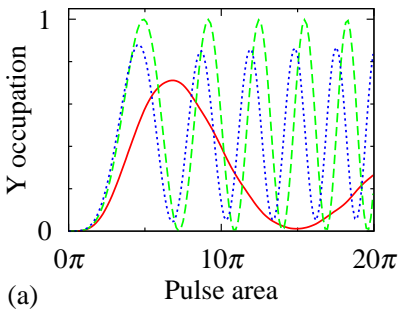

(a)

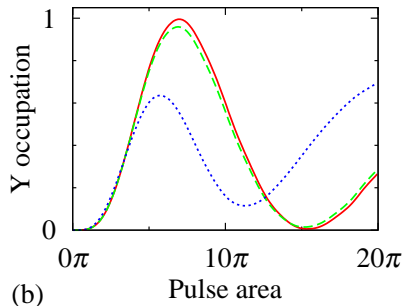

(b)
FIG. 11: (Color online) Dephasing of two-photon processes in a system with $E_{\mathrm{B}}>0$ for $\tau_{0}=5$ ps: (a) two-photon Rabi oscillations, starting from the ground state; (b) two-photon spin flip, starting from the $\left|\sigma_{+}\right\rangle$state. Red solid: $T=4 \mathrm{~K}$, green dashed: $T=10 \mathrm{~K}$, blue dotted: $T=40 \mathrm{~K}$.

fore contributing to dephasing even at low temperatures. This can be seen in Fig. 11(a), where the results of numerical simulations are plotted at a few values of temperature.

On the contrary, the positive biexciton shift is favorable for the spin flip process since now both the transitions to the ground and biexciton state are detuned down from the resonance. Hence, phonon-assisted transitions may be expected to contribute considerably only at high enough temperatures. This is indeed confirmed by the numerical solution to the TCL equation shown in Fig. 11(b), where it can be clearly seen that considerable dephasing occurs only when the temperature increases to a few tens of Kelvins.

One should note that the strong phonon-induced dephasing that affects the exciton spin flip process in the $E_{\mathrm{B}}<0$ case could be avoided by detuning the laser beam strongly above the resonance, as discussed in Sec. VB. However, strong dephasing of the biexciton Rabi oscillations in the present case cannot be dealt with in the same way because of the resonance condition required in this process.

\section{Exciton polarization rotation}

Let us now discuss the possibility of rotating the linear polarization of an exciton state via a two-photon process induced by a circularly polarized light. The energy diagram for this process is the same as that relevant to the spin flip and is shown in Fig. 1(b). As discussed in Sec. IIIB performing this two-photon transfer requires detuning off the two-photon biexciton resonance, i.e., $\Delta \neq E_{\mathrm{B}} / 2$. For a system with a bound biexciton $\left(E_{\mathrm{B}}<0\right)$, this means that the detuning between the laser frequency and the transition to either the ground state or the biexciton state decreases and enters the range of high spectral density of the phonon reservoir. This results in an increased contribution from phonon-assisted transitions and, therefore, in considerable damping of the polarization oscillations even at low temperatures,
FIG. 12: (Color online) Dephasing of two-photon polarization rotation for $\tau_{0}=5$ ps. (a) $E_{\mathrm{B}}=-4 \mathrm{meV}$, red solid line: $\Delta=-3 \mathrm{meV}, T=4 \mathrm{~K}$; green dashed line: $\Delta=2 \mathrm{meV}$, $T=4 \mathrm{~K}$; blue dotted line: $\Delta=2 \mathrm{meV}, T=40 \mathrm{~K}$. (b) $E_{\mathrm{B}}=4 \mathrm{meV}, \Delta=3 \mathrm{meV}$, red solid line: $T=4 \mathrm{~K}$; green dashed line: $T=10 \mathrm{~K}$; blue dotted line: $T=40 \mathrm{~K}$.

as shown in Fig. 12(a). Efficient polarization rotation is only possible for large positive detunings [see the diagram in Fig. 10(a)], for the same reasons as discussed in Sec. VB. Again, the quality of oscillations is reduced as soon as the temperature becomes comparable with the detuning from the ground state.

In a system with a positive biexciton shift, $E_{\mathrm{B}}>0$, phonon-induced transitions would require a phonon absorption and are therefore suppressed at low temperatures, as shown in Fig. 12(b) (red solid and green dashed lines). It should be noted, however, that the system evolution is relatively slow in this regime of operations since the detunings are close to the two-photon resonance, when the effect of the laser field completely vanishes (see Sec. IIIB). Therefore, large pulse intensities are needed to achieve the desired transformation of the system state. At higher temperatures [blue dotted line in Fig. 12(b)] the phonon absorption processes contribute considerably to the dephasing of the polarization rotation process.

\section{CONCLUSION}

Two-photon transitions driven by picosecond laser pulses detuned from both exciton and biexciton resonances in a quantum dot open the way to a rich variety of control schemes in a biexciton system. Using a linearly polarized laser field one can induce Rabi oscillations directly between the ground and biexciton state and coherently flip the circular polarization of an exciton state. The former involves a two-photon absorption or emission and requires exact two-photon resonance. The latter can be interpreted as 'photon exchange' (absorptionemission) and can be induced in a broad range of detunings. With a circularly polarized field, one can induce a rotation of the linear polarization of an exciton. This process takes place only off the two-photon resonance. The physics beyond these two-photon processes is highlighted by invoking the quantum-mechanical adiabatic theorem. If the individual excitons are treated as separate quantum 
subsystems (or qubits) then these two-photon control operations make it possible to prepare entangled states of these subsystems with a single laser pulse.

Already these results, restricted to the ideal evolution, confirm once more that the biexciton system is an interesting object of investigation from the point of view of quantum optics. They show that couplings between all four states forming the 'diamond' structure of the biexciton levels is essential for the system dynamics even if large detuning precludes real transitions between these levels. This suggests that models used for three-level atomic systems ('V-systems' ${ }^{\prime 11}$ ) may be insufficient for a description of QD systems.

In a real semiconductor system, the evolution is strongly affected by phonon-induced dephasing. In addition to the pure dephasing process appearing in an optically driven two-level exciton system, here we deal also with real phonon-assisted transitions to the states which, in the ideal case, should remain unoccupied. The effect of all the dephasing channels depends on the control scheme under consideration and on the energy level structure of the biexciton system. For negative biexciton shifts (bound biexcitons), the phonon-induced decoherence is relatively weak for two-photon Rabi oscillations and becomes stronger for the polarization flipping process. However, the additional freedom of detuning allows one to optimize the driving conditions against these decoherence effects also in the latter case so that spin flip fidelities as high as $10^{-3}$ are achievable. QDs with a positive biexciton shift are less suitable for these optical control schemes. In conrast, they are favorable for the twophoton rotation of the linear polarization: in this case, optimization of the control conditions against phononinduced dephasing can be much more efficient in a QD with an antibound biexciton than in a QD with a bound biexciton.

The study of phonon-induced dephasing shows, in addition, that a two-photon transition between the twosingle exciton states can be performed via states with constant average occupation of the QD. This eliminates the phonon response to the charge evolution and allows one to avoid the resulting dephasing. With this respect, the single-pulse two-photon control has a clear advantage over sequential transitions.

Out of the various processes discussed here, one (the biexciton oscillations) has already been observed experimentally ${ }^{20}$. The good agreement between the description based on the adiabatic theorem and the experimental results shows that the theory captures the essentials of the quantum evolution under actual laboratory conditions. The other processes take place under similar experimental conditions and therefore also seem to be experimentally feasible.
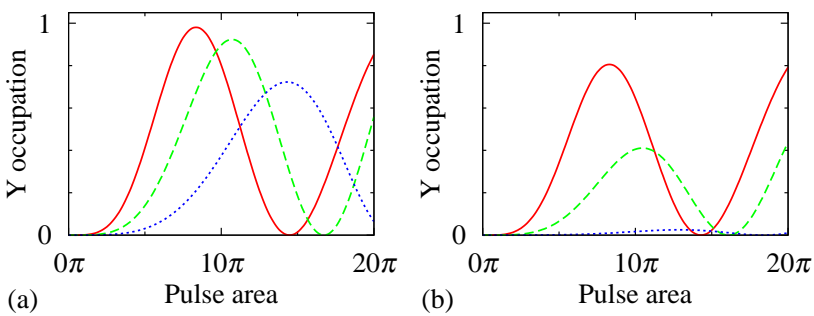

FIG. 13: (Color online) Rotation of the linear polarization of an exciton state with a circularly polarized pulse with the fine structure splitting of $\delta=30 \mu \mathrm{eV}$ (a) and $\delta=100 \mu \mathrm{eV}$ (b): the occupation of the $|\mathrm{Y}\rangle$ state as a function of the pulse area for $E_{\mathrm{B}}=-4 \mathrm{meV}$ and $\Delta=-3 \mathrm{meV}$; pulse durations $10 \mathrm{ps}$ (red solid lines), 20 ps (green dashed lines) and 40 ps (blue dotted lines).

\section{Acknowledgments}

The author is grateful to A. Grodecka for reading the manuscript and many helpful comments. This work was supported by Grant No. N202-071-32-1513 of the Polish MNiSW.

\section{APPENDIX A: FINE STRUCTURE SPLITTING}

In almost all QDs, electron-hole exchange interaction splits the single exciton states into a linearly polarized doublet of states $|\mathrm{X}\rangle$ and $|\mathrm{Y}\rangle$. The magnitude $\delta_{\mathrm{fs}}$ of this fine structure splitting can vary from several $\mu \mathrm{eV}$ to about $100 \mu \mathrm{eV}$. This splitting has no essential effect for the biexciton Rabi oscillations, while speaking of the exciton polarization flip makes sense only when the circularly polarized states can be considered well-defined, that is, when the total duration of the experiment does not exceed $\hbar / \delta_{\mathrm{fs}}$, which is of the order of 10 to 100 ps.

In this Appendix the effect of the fine structure splitting on the linear polarization rotation is discussed. The two linearly polarized states are well defined in the presence of this splitting. Due to the requirement for energy conservation, the fidelity of this type of coherent control depends on the relation between the pulse length and the magnitude of the splitting.

The additional contribution to the Hamiltonian, describing the fine structure splitting, is

$$
H_{\mathrm{fs}}=\frac{\delta_{\mathrm{fs}}}{2}(|\mathrm{X}\rangle\langle\mathrm{X}|-| \mathrm{Y}\rangle\langle\mathrm{Y}|)
$$

The results of simulations of the system dynamics (without dephasing) are presented in Fig. 13 for two values of the fine structure splitting $\delta_{\mathrm{fs}}$. If the pulse is long and, therefore, spectrally narrow, the transition between the two non-degenerate states becomes forbidden by energy conservation. As can be seen, for a typical value of $\delta_{\mathrm{fs}}=30 \mu \mathrm{eV}$, the effect of lifting the degeneracy be- 
comes important only for pulse durations of a few tens of picoseconds.
* Electronic address: Pawel.Machnikowski@pwr.wroc.pl

1 O. Benson, C. Santori, M. Pelton, and Y. Yamamoto, Phys. Rev. Lett. 84, 2513 (2000).

2 R. M. Stevenson, R. J. Young, P. Atkinson, K. Cooper, D. A. Ritchie, and A. J. Shields, Nature 439, 179 (2006).

3 A. Zrenner, E. Beham, S. Stufler, F. Findeis, M. Bichler, and G. Abstreiter, Nature 418, 612 (2002).

${ }^{4}$ X. Li, Y. Wu, D. Steel, D. Gammon, T. Stievater, D. Katzer, D. Park, C. Piermarocchi, and L. Sham, Science 301, 809 (2003).

5 D. Loss and D. P. DiVincenzo, Phys. Rev. A 57, 120 (1998).

6 E. Pazy, E. Biolatti, T. Calarco, I. D'Amico, P. Zanardi, F. Rossi, and P. Zoller, Europhys. Lett. 62, 175 (2003).

7 F. Troiani, E. Molinari, and U. Hohenester, Phys. Rev. Lett. 90, 206802 (2003).

8 S. E. Economou and T. L. Reinecke, Phys. Rev. Lett. 99, 217401 (2007).

9 E. M. Gauger, S. C. Benjamin, A. Nazir, and B. W. Lovett, Phys. Rev. B 77, 115322 (2008).

10 J. Berezovsky, M. H. Mikkelsen, N. G. Stoltz, L. A. Coldren, and D. D. Awschalom, Science 320, 349 (2008).

11 A. Kolli, B. W. Lovett, S. C. Benjamin, and T. M. Stace, Phys. Rev. Lett. 97, 250504 (2006).

12 E. M. Gauger, A. Nazir, S. C. Benjamin, T. M. Stace, and B. W. Lovett, New J. Phys. 10, 073016 (2008).

13 J. Förstner, C. Weber, J. Danckwerts, and A. Knorr, Phys. Rev. Lett. 91, 127401 (2003).

14 P. Machnikowski and L. Jacak, Phys. Rev. B 69, 193302 (2004).

15 A. Krügel, V. M. Axt, T. Kuhn, P. Machnikowski, and A. Vagov, Appl. Phys. B 81, 897 (2005).

16 T. Calarco, A. Datta, P. Fedichev, E. Pazy, and P. Zoller, Phys. Rev. A 68, 012310 (2003).

17 K. Roszak, A. Grodecka, P. Machnikowski, and T. Kuhn, Phys. Rev. B 71, 195333 (2005).

18 A. Grodecka, C. Weber, P. Machnikowski, and A. Knorr, Phys. Rev. B 76, 205305 (2007).

19 T. Flissikowski, A. Betke, I. A. Akimov, and F. Henneberger, Phys. Rev. Lett. 92, 227401 (2004).

20 S. Stufler, P. Machnikowski, P. Ester, M. Bichler, V. M. Axt, T. Kuhn, and A. Zrenner, Phys. Rev. B 73, 125304 (2006).

21 E. L. Ivchenko, Phys. Stat. Sol. (a) 164, 487 (1997).

${ }^{22}$ M. Bayer, G. Ortner, O. Stern, A. Kuther, A. A. Gorbunov, A. Forchel, P. Hawrylak, S. Fafard, K. Hinzer, T. L. Reinecke, S. N. Walck, J. P. Reithmaier, F. Klopf, and F. Schäfer, Phys. Rev. B 65, 195315 (2002).

23 A. J. Hudson, R. M. Stevenson, A. J. Bennett, R. J. Young, C. A. Nicoll, P. Atkinson, K. Cooper, D. A. Ritchie, and
A. J. Shields, Phys. Rev. Lett. 99, 266802 (2007).

${ }^{24}$ R. M. Stevenson, R. J. Young, P. See, D. G. Gevaux, K. Cooper, P. Atkinson, I. Farrer, D. A. Ritchie, and A. J. Shields, Phys. Rev. B 73, 033306 (2006).

25 B. D. Gerardot, S. Seidl, P. A. Dalgarno, R. J. Warburton, D. Granados, J. M. Garcia, K. Kowalik, O. Krebs, K. Karrai, A. Badolato, and P. M. Petroff, Appl. Phys. Lett. 90, 041101 (2007).

26 A. I. Tartakovskii, M. N. Makhonin, I. R. Sellers, J. Cahill, A. D. Andreev, D. M. Whittaker, J. P. R. Wells, A. M. Fox, D. J. Mowbray, M. S. Skolnick, K. M. Groom, M. J. Steer, H. Y. Liu, and M. Hopkinson, Phys. Rev. B 70, 193303 (2004).

27 W. Langbein, P. Borri, U. Woggon, V. Stavarache, D. Reuter, and A. D. Wieck, Phys. Rev. B 69, 161301(R) (2004).

28 B. Krummheuer, V. M. Axt, and T. Kuhn, Phys. Rev. B 65, 195313 (2002).

29 A. Grodecka and P. Machnikowski, Phys. Rev. B 73, 125306 (2006).

30 M. O. Scully and M. S. Zubairy, Quantum Optics (Cambridge University Press, Cambridge, 1997).

31 A. Messiah, Quantum Mechanics (North-Holland, Amsterdam, 1966).

32 P. Machnikowski, V. M. Axt, and T. Kuhn, Phys. Rev. A 75, 052330 (2007).

33 A. Vagov, M. D. Croitoru, V. M. Axt, T. Kuhn, and F. M. Peeters, Phys. Stat. Sol. (b) 243, 2233 (2006).

34 H.-P. Breuer and F. Petruccione, The Theory of Open Quantum Systems (Oxford University Press, Oxford, 2002).

${ }^{35}$ R. Alicki, M. Horodecki, P. Horodecki, and R. Horodecki, Phys. Rev. A 65, 062101 (2002).

36 R. Alicki, M. Horodecki, P. Horodecki, R. Horodecki, L. Jacak, and P. Machnikowski, Phys. Rev. A 70, 010501(R) (2004).

37 P. Machnikowski and L. Jacak, Acta Phys. Pol. 108, 761 (2005).

38 M. A. Nielsen and I. L. Chuang, Quantum Computation and Quantum Information (Cambridge University Press, Cambridge, 2000).

39 L. Jacak, P. Machnikowski, J. Krasnyj, and P. Zoller, Eur. Phys. J. D 22, 319 (2003).

${ }^{40}$ K. Roszak and P. Machnikowski, Phys. Lett. A 351, 251 (2006).

41 Q. Q. Wang, A. Muller, M. T. Cheng, H. J. Zhou, P. Bianucci, and C. K. Shih, Phys. Rev. Lett. 95, 187404 (2005). 